\title{
Evidências de validade entre a Escala de Depressão (EDEP), o BDI e o Inventário de Percepção de Suporte Familiar (IPSF)
}

\author{
Makilim Nunes Baptista ${ }^{1}$ - Universidade São Francisco \\ Mayra Silva de Souza - Universidade São Francisco \\ Gisele Aparecida da Silva Alves - Universidade São Francisco
}

\begin{abstract}
Resumo
A depressão é contemporaneamente definida como um transtorno de humor, marcada por um conjunto de sinais, sintomas, comportamentos e manisfestações fisiológicas apresentadas pelos indivíduos acometidos. Dentre os fatores relacionados com o desenvolvimento e manutenção da depressão, encontra-se o suporte familiar, entendido como manifestação de atenção, carinho, diálogo, liberdade, proximidade afetiva, autonomia e independência existente entre os integrantes da família. A presente pesquisa teve como objetivos a busca por evidências de validade para a Escala de Depressão (EDEP) baseada na relação com outras variáveis, avaliando o mesmo construto, ou seja, depressão sendo mensurada também pelo Inventário de Depressão de Beck - BDI e, ainda, avaliando construtos relacionados, sendo o suporte familiar mensurado pelo Inventário de Percepção de Suporte Familiar - IPSF. Participaram desta pesquisa 157 estudantes de uma universidade particular do sul de Minas Gerais, $75,5 \%$ do sexo feminino e $24,5 \%$ do sexo masculino, com idade média de 23,22 anos (DP=6,4), variando entre 18 e 51 anos. Nos resultados, encontraram-se correlações altas, positivas e significativas entre EDEP e BDI, conforme esperado, indicando que quanto maior a pontuação nas escalas, maior sintomatologia depressiva. Nas correlações entre a EDEP e as dimensões do IPSF, foram encontradas correlações negativas e significativas, sugerindo que quanto maior a sintomatologia de depressão, pior a percepção do suporte familiar recebido. Foram encontradas apenas diferenças marginalmente significativas entre os sexos, no que diz respeito à sintomatologia depressiva (EDEP e BDI), indicando que mulheres pontuariam mais nas escalas de depressão.

Palavras-chave: Sintomatologia depressiva; Família; Avaliação psicológica.
\end{abstract}

\section{Validity evidences between the Depression Scale (EDEP), the BDI and the Perception of Family Support Inventory (IPSF)}

\begin{abstract}
Depression is contemporary defined as a mood disorder, shown by a set of individual's signs, symptoms, behaviors and physiological manifestations. Among the related factors to developing and maintaining depression, there's family support, understood as manifestation of attention, care, dialog, freedom, affective proximity, autonomy and independence between family members. The present research aimed to search for validity evidences to Depression Scale (EDEP) based on the relation to other variables assessing the same construct, depression, being also assessed by Beck Depression Inventory - BDI, and assessing related constructs, family support, measured by Perception of Family Support Inventory - IPSF. 157 undergraduates from a private university located in south state of Minas Gerais, $75,5 \%$ from female gender and $24,5 \%$ from male gender, aging from 18 to 51 years old $(M=23,2 ; D P=6,4)$ took part in this study. At the results, high, significant and positive correlations were found between EDEP and BDI, as expected, indicating that the higher the scores on both scales, higher the depressive symptoms. As for the correlations between EDEP and IPSF dimensions, significant and negative correlations were found, suggesting that the higher depressive symptoms lower the perception of family support on individuals. Only marginally significant differences were found between genders, as to depressive symptoms (EDEP and BDI), indicating that women would score more at the depression scales.

Keywords: Depressive symptoms; Family; Psychological assessment.
\end{abstract}

\section{Introdução}

O termo depressão pode significar um sintoma que faz parte de inúmeros distúrbios emocionais sem ser exclusivo de nenhum deles, pode significar uma síndrome traduzida por muitos e variáveis sintomas ou, ainda, pode

1 Endereço para correspondência:

Programa de Pós-Graduação Strictu Sensu em Psicologia Apoio Executivo às Comissões de Pós Graduação

Av. Alexandre Rodrigues Barbosa, 45 - Centro, 13251-900, Itatiba-SP. Tel: (11) 4534-8040 significar uma doença. Enquanto sintoma, a depressão pode estar presente no transtorno de estresse pós-traumático, demência, esquizofrenia, doenças clínicas, alcoolismo e ainda, em circunstâncias econômicas e sociais adversas ou como resposta a eventos estressantes. Como síndrome, a depressão inclui não somente alterações de humor, tais como tristeza, apatia, falta de capacidade de sentir prazer, irritabilidade, mas compreende também alterações psicomotoras, cognitivas e vegetativas, como sono, apetite, etc. $\mathrm{Na}$ forma de doença, a depressão pode ser classificada de muitas formas, a saber, como 
transtorno depressivo maior, distimia, melancolia, depressão como parte da ciclotimia, depressão integrante do transtorno bipolar tipos I e II, dentre outros (Del Porto, 2002).

$\mathrm{O}$ Diagnostic and statistical manual of mental disorders [DSM-IV] (APA, 2002), estabelece critérios para o episódio e para o transtorno depressivo maior, dentre os quais é necessária a identificação de cinco ou mais sintomas, incluindo ao menos humor depressivo (que em crianças e adolescentes, pode ser irritável) e/ou perda de interesse ou prazer, que perdurem no mínimo duas semanas, representando uma alteração diante do funcionamento anterior. Outros sintomas importantes para a classificação do transtorno depressivo maior são: a perda ou ganho significativo de peso sem estar em dieta, ou diminuição ou aumento do apetite, insônia ou hipersonia, agitação ou retardo psicomotor, fadiga ou perda de energia, sentimento de inutilidade ou culpa excessiva ou inadequada (que pode ser delirante e não meramente auto-recriminação ou culpa por estar doente), capacidade diminuída de pensar ou concentrar-se, ou indecisão, esses sintomas apresentados por quase todos os dias, pensamentos de morte recorrentes (não apenas medo de morrer), ideação suicida recorrente sem um plano específico e tentativa de suicídio ou plano específico para cometer suicídio. Em adição, indivíduos com episódio depressivo maior apresentam, com freqüência, propensão ao choro, ansiedade, fobias, irritabilidade, ruminação obsessiva, preocupação com a saúde física e queixas de dores.

Considera-se ainda se os sintomas causam sofrimento clinicamente significativo ou prejuízo em áreas importantes da vida do indivíduo, tais como social, afetiva, ocupacional, etc. se eles não se devem aos efeitos fisiológicos diretos de uma substância ou de uma condição médica geral, se mais bem não são explicados por condição de luto (perda de um ente querido) e se persistem por mais de 2 meses ou são caracterizados por acentuado prejuízo funcional, preocupação mórbida com desvalia, ideação suicida, sintomas psicóticos ou retardo psicomotor (APA, 2002).

De acordo com Grevet e Knijnik (2001), os sintomas da depressão classificam-se em alterações de humor (triste ou deprimido), alterações psicomotoras (agitação ou retardo), cognitivas (alterações subjetivas da memória, atenção e velocidade do pensamento e do raciocínio) e neurovegetativas (alterações de peso, sono, interesse sexual e sintomas somáticos, como fraqueza muscular, peso na região das costas, e cefaléia). Podem ainda classificar-se nos ritmos circadianos (alterações nos ritmos de sono-vigília, liberação de cortisol e regulação da temperatura) e sazonalidade (padrão caracterizado pelo aumento de episódios depressivos em determinadas épocas do ano).

Vários fatores encontram-se relacionados com a depressão, como por exemplo idade e sexo. A depressão pode afetar as pessoas em qualquer fase da vida, porém a incidência mais alta é nas idades médias e há indícios de crescimento desse transtorno durante a adolescência e no início da vida adulta. Ainda, essa desordem é mais comum no sexo feminino, estimando-se prevalência do episódio depressivo em $1,9 \%$ no sexo masculino e $3,2 \%$ no feminino (OMS, 2001).

Os transtornos depressivos causam muito sofrimento, além de acometerem os indivíduos na vida ocupacional, uma vez que a depressão crônica provoca maior incapacidade para o trabalho, perdendo apenas para o infarto do miocárdio (OMS, 2001). Ainda, a depressão apresenta alta comorbidade com outros quadros psiquiátricos e também com doenças físicas, sendo difícil a detecção de um quadro depressivo (Grevet \& Knijnik, 2001).

Existem vários instrumentos que avaliam depressão, bastante utilizados em pesquisas mundiais, tais como a Escala de Depressão de Hamilton (HDRS), a Montgomery Asberg Depression Scale - MADS, a Escala de Rastreamento Populacional para Depressão (Center for Epidemiologic Studies Depression Scale - CES-D), e a Escala de Depressão de Beck - BDI. Essa última escala foi escolhida para ser utilizada no presente estudo por ser auto-aplicativa e por apresentar boa qualidade psicométrica confirmada por vários estudos de validade, inclusive no Brasil (Cunha, 2001; Goreinstein, Andrade \& Zuardi, 2000; Santor, Gregus \& Welch, 2006).

A validade de um teste ou prova diz respeito à eficiência em avaliar o que se deseja. Dessa forma uma evidência de validade é encontrada quando as interpretações dos resultados de um teste alcançam determinado nível de sustentação em constatações teóricas ou empíricas. É possível então encontrar evidências de validade para situações diversas, podendo implicar tipos específicos de uso do teste avaliado. Quando essas evidências não são encontradas não há garantia de que as interpretações sugeridas sejam autênticas, pois não é possível assegurar o 
que o instrumento realmente avalia (AERA, APA \& NCME, 1999).

Existem cinco classificações para as evidências de validade de testes buscadas em pesquisas, sejam quais forem suas bases: o conteúdo, as relações com outras variáveis, a estrutura interna, o processo de resposta e as conseqüências da testagem. Destaca-se a evidência de validade baseada na relação com outras variáveis, que sustentará os objetivos deste trabalho. Esse tipo de validade procura por padrões de correlação entre os escores do teste e outras variáveis que meçam o mesmo construto, construtos relacionados ou com variáveis que meçam construtos diferentes (AERA, APA \& NCME, 1999).

Nesse sentido, Prieto e Muniz (2000) descrevem um modelo para avaliar a qualidade dos testes psicológicos, baseados em critérios psicométricos internacionais. Algumas informações que os autores sugerem estão relacionadas com a área a qual pertence o teste, o formato dos itens (tipo Likert, dicotômico, etc.), número de itens, tipo de administração (oral, papel), qualificação requerida para ser respondente do teste, descrição das populações em que o teste foi aplicado e avaliado, procedimentos de correção, forma de aplicação, tempo gasto para responder ao teste, fundamentação teórica, qualidade das instruções, análise dos itens e informações psicométricas dos dados de estudos de validade.

Em relação aos dados de validade, Prieto e Muniz (2000) se referem a valores das correlações esperadas no caso de estudos com instrumentos similares, como, por exemplo, duas escalas que se proponham a medir sintomatologia de depressão. Os valores de correlação considerados excelentes, dependendo do tamanho das amostras, são a partir de 0,60, o que indicaria que os instrumentos realmente mensuram o mesmo construto, ou construtos similares, no entanto não há um critério préestabelecido para construtos relacionados, como no caso de sintomas de depressão e suporte familiar.

Em um estudo de revisão, Baptista, Baptista e Dias (2001), analisaram a estrutura e o suporte familiar como fatores de risco para a depressão em adolescentes. Os autores ressaltam que não se pode pensar em uma causa específica para o desenvolvimento da depressão, que é multifacetária, sendo influenciada por fatores biológicos/ genéticos, psicológicos e sociais. Porém, as rápidas mudanças sociais familiares, no que tange às mudanças na composição, estrutura física e conseqüentemente nas regras e papéis da família, acabam por colaborar com a prevalência de depressão na população adolescente. A família ainda deve ser considerada como amortecedora diante dos eventos estressantes, enfrentados no cotidiano de adolescentes.

O grupo familiar, na cultura ocidental, constitui-se de agrupamento de marido, esposa e filhos, e tem como função não apenas a mediação entre indivíduo e sociedade, sendo apontado como essencial para a sobrevivência e desenvolvimento dos membros da família. Assim, além da socialização, a família exerce papéis de proteção, afeição, provimento de recursos financeiros, transmissão de valores éticos, morais e culturais (Alchaer, Bahsas, Nieto \& Salinas, 1994; Campos, 2004; Carvalho \& Almeida, 2003).

A família pode ser considerada o alicerce da construção de um modelo relacional que permite aos seus integrantes a criação de outras relações, desde as laborais, de amizade, de parentesco, até a construção de uma nova família. Pautas, regras, papéis e obrigações são assimilados em cada um de seus membros que, por oposição ou similaridade, se identificam com seu grupo familiar. Tais identificações não implicam somente à construção cognitiva e de personalidade, ou seja, não somente demarcam fronteiras de estilos de personalidade, mas também reproduzem pautas de interação, alternativas relacionais e formas de manifestar afetos e emoções (Ceberio, 2006).

$\mathrm{Na}$ dinâmica de uma família funcional são indispensáveis: comunicação congruente, direcional, funcional e com carga emocional; regras coerentes e flexíveis, porém com limites estabelecidos; liderança compartilhada com filhos de forma democrática; auto-estima e autoconfiança, na medida em que autonomia e proteção são oferecidas equilibradamente; e relação conjugal integrada, de forma que possibilite à família atuar como um todo, preservando a individualidade de cada um. A percepção dessa dinâmica pelos integrantes da família é que lhe concede recursos necessários ao crescimento individual e apoio diante das dificuldades da vida ou doenças intercorrentes (Féres-Carneiro, 1992; Lidchi \& Eisenstein, 2004).

Compreendendo as funções e dinâmica familiar e a importância destas no desenvolvimento dos integrantes da família, suas influências tanto podem ser positivas como negativas. Para Féres-Carneiro (1992), a família pode funcionar como facilitadora da saúde 
emocional de seus membros quando cada um desses conhece e desempenha um papel específico. Esses papéis, por sua vez, precisam estar bem definidos, adequados e flexíveis. Quando a família desempenha esse papel facilitador, ela passa a funcionar como um sistema de suporte para seus membros.

O suporte familiar é um construto de difícil definição e operacionalização, e abrange questões relativas às complexas relações que se estabelecem entre os membros de uma família. Baptista e Oliveira (2004) entendem suporte familiar como manifestação de atenção, carinho, diálogo, liberdade, proximidade afetiva, autonomia e independência existente entre os integrantes da família (características psicológicas) e, dessa forma, diferenciam-no do conceito de estrutura familiar referente ao número de pessoas pertencentes a uma família, sua disposição e composição (características físicas). Para Campos (2004), a percepção da pessoa que recebe o apoio da família e o considera satisfatório, se configura no principal efeito do suporte, pois se sentindo amado, valorizado, compreendido, reconhecido, acolhido, protegido, cuidado e compartilhando de uma rede de recursos e informações, o indivíduo torna-se mais resistente para enfrentar o ambiente, trazendo conseqüências positivas para seu bem estar, podendo reduzir o estresse, aumentar a auto-estima e o bem-estar psicológico.

A família assume um papel particularmente importante em fornecer oportunidades de suporte para as pessoas jovens. Entretanto, uma família suportiva se reflete não apenas pelo grau de ligação social familiar, mas também pelas oportunidades que a mesma oferece quanto aos seus suportes dentro da comunidade. $\mathrm{O}$ acesso limitado às relações suportivas dentro da família, combinado com o envolvimento restrito com os suportes da comunidade, tem sido associado especificamente aos jovens que são deprimidos (Ostrander, Weinfurt \& Nay, 1998).

Um estudo realizado por Martin e Waite (1994) investigou a relação entre ideação, intenção suicida, depressão e vínculo com os pais. Participaram dessa pesquisa 681 estudantes com idade média de 15 anos, e os instrumentos utilizados foram o Parental Bonding Instrument (PBI), que avalia o suporte familiar, especificamente carinho e proteção, e o Youth Self Report (YSR), que avalia ideação e intenção suicida e depressão. Foram encontradas diferenças significativas nas subescalas do PBI para os grupos de casos e não casos para depressão, ideação/intenção suicida.
Ainda foram encontradas correlações significantes entre a subescala de depressão do YSR e o PBI, de forma que, para os homens, os escores de carinho materno e paterno apresentaram as respectivas correlações $r=-0,31$ e $r=-0,26$ e para proteção materna e paterna, $r=0,32$ e $r=0,23$, e para as mulheres, os escores de carinho materno e paterno apresentaram correlações $r=-0,35$ e $r=-0,40$, respectivamente e para proteção materna e paterna $r=0,30$ e $r=0,24$. Esses resultados sugerem que baixo carinho e alta proteção são associados com o aumento dos níveis de depressão.

Em um estudo de correlação, Baptista e Oliveira (2004) avaliaram a sintomatologia de depressão e o suporte familiar em adolescentes. Participaram da pesquisa 154 adolescentes do ensino médio de uma escola pública. Foi utilizado um questionário de caracterização, contendo fatores de risco para depressão, além do Inventário de Depressão Infantil (CDI) e o Parental Bonding Instrument (PBI). Como resultado, o suporte familiar esteve negativamente avaliado no grupo com sintomatologia depressiva, indicando que, quanto maior a sintomatologia depressiva no adolescente, mais inadequada é a sua percepção do suporte familiar recebido. Os autores ainda relatam que os sintomas de depressão também podem estar envolvidos na percepção do suporte familiar, já que indivíduos com depressão podem tender a distorcer negativamente o apoio provindo da família e de amigos.

Parker, Tupling e Brown (1979) postularam que os pais carinhosos e empáticos permitem que o filho tenha uma maior auto-estima, o que proporciona proteção contra a depressão na fase adulta. Os autores explicam que a superproteção está relacionada à depressão por meio da inibição da autonomia e da competência social. Ao desencorajar a independência e nutrir uma expectativa de que as conseqüências não dependem de respostas, o abandono e a depressão são providos quando são enfrentados os fatores de estresse.

Nesta mesma perspectiva, utilizando também o Parental Bonding Instrument (PBI) e suas subescalas que avaliam cuidado e proteção dos pais, pacientes de dois tipos de desordem depressiva foram comparados com um grupo controle. Tanto os pacientes com desordens maníaco-depressivas como os neuróticos depressivos relataram baixo cuidado dos pais e grande superproteção materna. Experiências depressivas no grupo não-clínico foram negativamente associadas com baixo cuidado dos 
pais e fracamente associada com superproteção dos pais (Parker, 1979).

$\mathrm{O}$ relacionamento que os pais ou cuidadores estabelecem com a criança durante a infância é de suma importância. O afeto, a atenção e o cuidado constante dispensados permitem que a criança se desenvolva com menores probabilidades de desenvolver transtornos mentais. Já a nãotransmissão desses cuidados pode aumentar a probabilidade dessa criança e manifestar distúrbios mentais e comportamentais, tanto durante a infância quanto em outras fases posteriores da vida (OMS, 2001).

Quanto mais resistente for o indivíduo perante aos eventos estressantes da vida, mais ele estará associado às características de afeto recebido no início de sua vida. O afeto é construído na memória da criança e pode protegêla dos estressores internos e externos. O afeto inseguro é uma vulnerabilidade central para a criança que cresce e pode levar também a problemas sociais. Além da resiliência, o afeto inicial na vida fornece uma base para o domínio das tarefas de desenvolvimento e a competência nas relações entre os amigos, trabalho acadêmico e autocontrole (Weinman, Buzi, Smith \& Mumford, 2003).

Em outra perspectiva, que envolve perda dos pais enquanto perda de fonte de suporte familiar, Patten (1991) reuniu dados de 2.432 sujeitos em uma metanálise, encontrando que, em relação ao sexo feminino, houve uma significativa associação entre a perda de um dos pais antes dos 11 anos e depressão; e que perder a mãe mais cedo ainda poderia representar um duplo risco para depressão. Houve uma prevalência significativamente mais alta de perda na infância entre os indivíduos com depressão do que entre os indivíduos que não sofriam de depressão.

Bron, Strack e Rudolph (1991) estudaram 328 pacientes com depressão, desordem distímica ou desordem de ajuste com humor deprimido. Eles foram questionados sobre perdas sofridas na infância por morte de um ou ambos os pais e também por divórcio dos pais com pelo menos um ano de separação. Não houve estatísticas significativas entre experiências de perda na infância e tipo de depressão, sexo e idade, porém foi encontrado um aumento na prevalência de tentativas de suicídio naqueles pacientes com experiências de perdas na infância, tanto por separação quanto por morte, sendo que o aumento da tendência suicida foi atribuído principalmente à perda do pai.
Luecken (2000) estudou a associação entre perda por morte de um dos pais, sentimentos de hostilidade, depressão, suporte social e qualidade das relações familiares em adolescentes. Participaram dessa pesquisa 30 estudantes universitários que experimentaram a morte de um dos pais antes dos 16 anos e 31 estudantes em condição controle. Foram utilizados questionários para medir o suporte social (The Interpersonal Support Evaluation), a hostilidade (Cook Medley Hostility Scale), a depressão (Beck Depression Inventory) e a qualidade das relações familiares (Moos Family Environment Scale). Apesar da baixa amostragem, aqueles com perda de um dos pais apresentaram altos escores de hostilidade, maior intensidade de sintomas depressivos e menor suporte social apenas quando apresentavam pobres relações familiares atuais.

Sendo assim, constitui-se como objetivo deste estudo a busca por evidências de validade para um novo conjunto de itens que aborda a sintomatologia depressiva, que se encontra em desenvolvimento, intitulado EDEP (Escala de Depressão) (Baptista \& Sisto, em desenvolvimento). Espera-se uma relação convergente entre a EDEP e o BDI, já que consideram o mesmo construto (depressão), e entre a EDEP e o IPSF (Inventário de Percepção de Suporte Familiar), que abordam construtos relacionados (depressão e suporte familiar), conforme aponta a literatura estudada. Como objetivos secundários, serão buscadas diferenças entre sexos de acordo com a sintomatologia depressiva pontuada pelos participantes.

\section{Método}

\section{Participantes}

Participaram desta pesquisa 167 estudantes de uma universidade particular de uma cidade do sul de Minas Gerais. Desse total, 10 participantes foram excluídos porque deixaram de responder mais de $10 \%$ de algum dos instrumentos utilizados na pesquisa, o que correspondeu a uma perda amostral de $0,6 \%$, totalizando ao final 157 estudantes. Destes $75,5 \%$ eram do sexo feminino e $24,5 \%$ do sexo masculino, com idade média de 23,22 (desvio padrão de 6,4), variando entre 18 e 51 anos.

\section{Instrumentos}

Escala de Depressão (EDEP) (Baptista \& Sisto, 2007); como será intitulada, é inicialmente um conjunto de descritores de sintomas de 
depressão, baseado nos indicadores retirados do DSM-IV-TR (APA, 2002), CID-10 (OMS, 2000), Terapia Cognitiva da Depressão (Beck, Rush, Shaw \& Emery, 1982) e Princípios do Comportamento (Ferster, Culbertson \& Boren, 1977), resultando inicialmente em um screening (rastreamento) de sintomas de depressão. Para sua construção, foram elaboradas frases apontando extremos (frases negativas e positivas), para que o sujeito indicasse, em uma escala, como se sentia em relação aos descritores. Essas frases foram elaboradas para cada um dos indicadores de depressão, quais sejam: humor deprimido, perda ou diminuição de prazer, choro, desesperança, desamparo, indecisão, sentimento de incapacidade, sentimentos de inadequação, carência/dependência, negativismo, esquiva de situações sociais, queda de produtividade, inutilidade, autocrítica exacerbada, culpa, diminuição de concentração, pensamento de morte, auto-estima rebaixada, falta de perspectiva sobre o presente, falta de perspectiva sobre o futuro (incorporado à desesperança), hipocondria, alteração de apetite, alteração de peso, insônia / hipersonia, lentidão / agitação psicomotora, perda de libido, fadiga / perda de energia e irritação. Após a eliminação de frases repetidas, dúbias e a adaptação ao tempo verbal, o conjunto inicial constou de 75 questões e teve como opções de resposta uma escala Likert de cinco pontos, caracterizadas por cinco círculos entre as duas extremidades do item (que era composto de duas frases dicotômicas), na qual o indivíduo deveria responder como se sentia em relação às situações propostas, como nos exemplos, "Tenho chorado muito - Não Tenho chorado; O futuro será melhor - Não acredito que as coisas melhorem; Tenho dormido como antes - Está sendo difícil pegar no sono; Sinto prazer em realizar minhas atividades As coisas não me agradam como antigamente; Sinto-me cada vez mais sozinho - Não sinto solidão".

Inventário de Depressão de Beck (BDI) (Cunha, 2001): é um instrumento para a medida de sintomatologia de depressão muito utilizado na área clínica e em pesquisas, e que possui vários estudos psicométricos com amostras brasileiras. É uma escala de auto-relato composta por 21 itens, cada um com quatro alternativas com escores de 0 a três, que subentendem graus crescentes da depressão. Os 21 itens referem-se a tristeza, pessimismo, sentimento de fracasso, insatisfação, punição, auto-aversão, idéias suicidas, choro, irritabilidade, retraimento social, indecisão, mudança na auto-imagem, dificuldade de trabalhar, insônia, fatigabilidade, perda de apetite e peso, preocupações somáticas e perda da libido. O escore total permite a classificação dos níveis de intensidade da depressão, que varia entre depressão mínima (0-9), depressão leve (10-16), depressão moderada (17-29) e depressão severa (30-63).

Inventário de Percepção do Suporte Familiar (IPSF) (Baptista, 2005; 2007) - este instrumento avalia a percepção do indivíduo ante o suporte que recebe da sua família. Consta de 42 questões, com três opções de respostas, quais sejam "sempre", "às vezes", e "nunca". Possui três dimensões, que seguem: 1) afetivo-consistente: essa dimensão contém 21 itens e evidencia as relações afetivas positivas intra-familiares, desde o interesse pelo outro, até a expressão verbal e nãoverbal de carinho, clareza nos papéis e regras dos integrantes da família, bem como a habilidade nas estratégias de enfrentamento de situaçõesproblema; 2) adaptação familiar: com um total de 13 itens que expressam sentimentos negativos em relação à família, como isolamento, exclusão, raiva, vergonha, relações agressivas de brigas e gritos, irritação, incompreensão e, ainda, percepção de relações de competição na família, interesse e culpabilidade entre os membros em situações de conflito. Os itens deste fator foram invertidos para que pudessem ser calculados com valência igual aos itens das outras duas dimensões; e 3) autonomia familiar: composto por 8 itens, demonstra a percepção de autonomia que o indivíduo tem de sua família, o que denota relações de confiança, privacidade e liberdade entre os membros da família. Já foram encontradas evidências de validade de construto e critério para o inventário, tais como os estudos de Santos (2005), Rigotto (2006), Aquino (2007), Souza (2007) e Baptista, Santos e Alves (2008).

\section{Procedimento}

Após a aprovação deste projeto por Comitê de Ética em Pesquisa, buscou-se o consentimento dos universitários que participaram da pesquisa mediante Termo de Consentimento Livre e Esclarecido (TCLE), impresso em duas vias, no qual constam todas as informações pertinentes à pesquisa em questão. A aplicação dos instrumentos se deu de forma coletiva, em horário de aula. Foi entregue um caderno contendo as duas vias do TCLE, seguido dos instrumentos: Escala de Depressão (EDEP), Inventário de Depressão de Beck (BDI) e Inventário de 
Percepção de Suporte Familiar (IPSF). Em média, a aplicação durou uma hora.

\section{Resultados e discussão}

Para análise dos dados utilizaram-se testes não-paramétrico que seguem: a) o coeficiente de correlação de Spearman $\left(r_{s}\right)$ para correlacionar os escores dos testes e dimensões; b) o teste $U$ de Mann-Whitney, para buscar diferenças entre os sexos. O nível de significância adotado para tais análises foi o de $\mathrm{p} \leq 0,05$.

$\mathrm{Na}$ correlação entre EDEP e BDI, como esperado, enquanto itens que avaliam os sintomas de depressão, os escores caminharam no mesmo sentido, indicando que quanto maior a pontuação nas escalas, maior sintomatologia depressiva. Conforme é possível notar na Tabela 1, a correlação encontrada entre os escores desses dois instrumentos é alta e significativa $\left(r_{s}=0,75\right.$; $p=0,000)$, atendendo assim às expectativas do estudo quanto a evidência de validade para a EDEP, baseada na relação com outras variáveis medindo o mesmo construto, no caso, a depressão. Nesse sentido Prieto e Muniz (2000) consideram que correlações acima de 0,60 para instrumentos que avaliam construtos similares já podem ser consideradas excelente, o que ocorreu neste estudo, mesmo apesar da baixa amostragem utilizada.

Tabela 1 - Correlações da EDEP com BDI e IPSF

\begin{tabular}{ccccccc}
\hline & BDI & $\begin{array}{c}\text { IPSF } \\
\text { Afetivo-consistente }\end{array}$ & $\begin{array}{c}\text { IPSF } \\
\text { Adaptação familiar }\end{array}$ & $\begin{array}{c}\text { IPSF } \\
\text { Autonomia familiar }\end{array}$ & $\begin{array}{c}\text { IPSF } \\
\text { Suporte total }\end{array}$ \\
\hline EDEP & $r$ & 0,75 & $-0,36$ & $-0,37$ & $-0,32$ & $-0,42$ \\
& $p$ & 0,000 & 0,000 & 0,000 & 0,000 & 0,000 \\
\hline
\end{tabular}

Nas correlações entre a EDEP e as dimensões do IPSF, os resultados apontam para uma associação negativa, na medida em que, quanto maior o escore na Escala de Depressão, maior a sintomatologia, e quanto maior a pontuação no Inventário de Percepção de Suporte Familiar, melhor o suporte familiar percebido. Não se esperavam correlações altas, uma vez que os construtos envolvidos estão relacionados, porém são diferentes; e como esperado, as correlações foram negativas moderadas (Tabela 1). Esse resultado sugere que quanto maior a sintomatologia de depressão, menor o suporte familiar percebido.

As correlações envolvendo as dimensões do IPSF sugerem que quanto maior a percepção de afeto, carinho, interesse, atenção, diálogo, inclusão, compreensão, clareza de papéis e regras familiares, independência e habilidade na resolução de situações-problema, menor a sintomatologia depressiva apresentada pelos participantes. Consoante aos resultados da pesquisa atual, o estudo de Martin e Waite (1994) aponta como risco para depressão, o baixo carinho e alta proteção dos pais. Os autores encontraram correlações parecidas com as do estudo em questão, quais sejam, $r=-0,31$ e $r=-0,26$ (respostas de homens para carinho materno e paterno correlacionadas com depressão) e $r=-0,35$ e $r=$ 0,40 (respostas de mulheres para carinho materno e paterno correlacionadas com depressão), assim como no presente estudo encontrou-se $r=0,36$ na correlação entre EDEP e a dimensão afetivoconsistente do IPSF, que denota relações afetivas de carinho entre os membros da família; $r=0,32$ e $r=0,23$, (respostas de homens para proteção materna e paterna correlacionadas com depressão) e $r=0,30$ e $r=0,24$ (respostas de mulheres para proteção materna e paterna correlacionadas com depressão), e na pesquisa em questão encontrou-se $r=0,32$ na correlação entre EDEP e a dimensão autonomia familiar do IPSF, que evidencia relações de independência, contrárias as relações de superproteção.

Tratando-se ainda deste resultado último, Parker, Tupling e Brown (1979) explicaram que a depressão deriva do desencorajamento dos pais à independência dos filhos, quando esses enfrentam situações estressoras. Corrobora também o estudo de Parker (1979), no qual pacientes depressivos e maníaco-depressivos relataram baixo cuidado dos pais e grande superproteção materna.

Como apontou a correlação da EDEP com a dimensão adaptação familiar do IPSF, quanto mais sentimentos positivos em relação à família, compreensão, inclusão, menor a sintomatologia depressiva. Parker, Tupling e Brown (1979) consideraram que os pais empáticos permitem que os filhos tenham auto-estima, o que resulta em proteção contra a depressão na fase adulta.

Confirmando os resultados da pesquisa, Baptista, Baptista e Dias (2001) compreendem a 
família como fonte amortecedora do estresse e consideram a falta de suporte familiar como um fator de risco para a depressão. No estudo de Baptista e Oliveira (2004), o suporte familiar esteve negativamente associado com a sintomatologia depressiva, assim como no presente estudo, da mesma forma que no estudo de Dias (2008), que utilizou a EDEP e o IPSF em pacientes de um grupo clínico (doenças inflamatórias intestinais) e não clínico (acompanhantes) de um hospital escola, encontrando correlações que variaram entre $-0,37$ do EDEP com a dimensão afetivo-consistente a $0,51 \mathrm{com}$ as pontuações totais do IPSF, muito próximas às encontradas na atual pesquisa.

Não foram encontradas diferenças significativas entre os sexos, no que diz respeito à sintomatologia depressiva (EDEP e BDI), e percepção de suporte familiar (IPSF). Os resultados apontam para uma maior pontuação para mulheres na EDEP ( $U=1963,000$ e $p=0,279)$ e no BDI $(U=1806,000$ e $p=0,082)$, porém esses resultados não são significativos. Para a OMS (2001), as mulheres são mais suscetíveis à depressão que os homens, no entanto não foi observado nesta amostra, apesar de ter sido observada uma tendência significativa no BDI.

O estudo em questão se propôs a buscar evidências de validade para a Escala de Depressão (EDEP), procurando relações com outras variáveis, quais sejam, a depressão (mesmo construto) e o suporte familiar (construto relacionado), encontrando resultados desejáveis para esta medida. Trata-se de um conjunto de descritores prático, auto-aplicável e satisfatório para rastrear sintomas de depressão, podendo ser uma ferramenta no auxílio no diagnóstico de tal desordem. Sendo assim, este conjunto de descritores, chamados inicialmente de escala de Depressão (EDEP), mostrou-se bastante promissor para a avaliação da sintomatologia depressiva, já que foi observada alta correlação com um dos instrumentos mais utilizados para avaliar depressão, no caso o BDI, inclusive validado para a população brasileira (Cunha, 2001; Santor, Gregus \& Welch, 2006). A partir do momento em que haja amostras específicas de pacientes diagnosticados com depressão, ou mesmo com sintomatologia clinicamente significativa, já é possível desenvolver estudos de validade de construto com a EDEP, possibilitando replicar o presente estudo.

Constituem limitações a amostra restrita, assim como a pouca variabilidade entre os sexos (predominância de mulheres). Portanto, ainda é pertinente estudar as relações aqui tratadas com amostras maiores, para que os resultados sejam comparados e avaliados. Sugere-se também estudar a EDEP com outras variáveis relacionadas, tais como estratégias de enfrentamento (coping), suicídio, ansiedade, desesperança, eventos estressantes de vida, dentre outros, incluindo também variáveis sócio-demográficas como nível socioeconômico, estado civil, raça e escolaridade.

\section{Referências}

Alchaer, J. R. A., Bahsas, F. B., Nieto, R. H. \& Salinas, P. J. (1994). Relacion entre el funcionalismo familiar, el estrés y la ansiedad. Revista de la Facultad de Medicina, 3 (3-4), 81-86.

American Educational Research Association AERA, American Psychological Association APA, Nacional Council on Measurement in Education - NCME (1999). Standards for Educational and Psychological Testing. Washington, DC: American Educational Research Association.

APA - American Psychiatric Association. (2002). Diagnostic and Statistical Manual of Mental Disorders-IV-TR. Washington, DC.

Aquino, R. R. (2007). Inventário de Percepção de Suporte Familiar (IPSF) e Escala de Vulnerabilidade ao Estresse no Trabalho (EVENT): evidência de validade. Dissertação de Mestrado. Itatiba: Psicologia. Programa de Pós-Graduação Stricto-Sensu em Psicologia da Universidade São Francisco.

Baptista, M. N. (2005). Desenvolvimento do Inventário de Percepção de Suporte Familiar (IPSF): estudos psicométricos preliminares, Psico-USF, 10, (1), 11-19.

Baptista, M. N., Baptista, A. S. D., \& Dias, R. R. (2001). Estrutura e Suporte Familiar como Fatores de Risco na Depressão de Adolescentes. Psicologia Ciência e Profissão, 21 (2), 52-61.

Baptista, M. N. \& Oliveira, A. A. (2004). Sintomatologia de depressão e suporte familiar em adolescentes: um estudo de correlação. Revista Brasileira de Crescimento e Desenvolvimento Humano, 14 (3), 58-67.

Baptista, M. N. (2007). Inventário de percepção de suporte familiar (IPSF): estudo componencial 
em duas configurações. Psicologia ciência e profissão, 27(3), 496-509.

Baptista, M. N., Santos, T. M. M, \&Alves, G. A. S. (2008). Suporte familiar, auto-eficácia e lócus de controle: evidências de validade entre os construtos. Psicologia Ciência e Profissão, 28(3), $260-271$.

Baptista, M. N. \& Sisto, F. F. (2007). Escala de Vulnerabilidade ao Estresse no Trabalho EVENT. Manual teórico não publicado.

Beck, A. T., Rush, A. J., Shaw, B. F. \& Emery, G. (1982). Uma visão geral. Em A. T. Beck, A. J. Rush, B. F. Shaw \& G. Emery, Terapia cognitiva da depressão (pp. 15-45). Rio de Janeiro: Zahar.

Bron, B., Strack, M., \& Rudolph, G. (1991). Childhood experience of loss and suicide attempts: Significance in depressive states of major depressed and dystimic or adjustment disorder patients. Journal Affective Disorders, 23, 165-72.

Campos, E. P. (2004). Suporte social e família. Em J. Mello Filho, Doença e Família (pp. 141-161). São Paulo: Casa do Psicólogo.

Carvalho, I. M. M. de \& Almeida, P. H. de. (2003). Família e proteção social. São Paulo em Perspectiva, 17 (2), 109-122.

Ceberio, M. R. (2006). Vejas y nuevas famílias: La transición hacia nuevas estructuras familiares. Interpsiquis. Obtido em fevereiro 2006, em World Wide Web: http//:www.psiquiatria.com.

Cunha, J. (2001). Manual em português das Escalas Beck. São Paulo: Casa do Psicólogo.

Del Porto (2002). Depressão: conceito e diagnóstico. Em J. J. Mari e cols. (2002). Atualização em Psiquiatria 1. (pp. 21-29). São Paulo: Casa do Psicólogo.

Dias, C. C. (2008). Evidências de Validade Convergente e Discriminante da Escala de Depressão (EDEP). Dissertação de Mestrado. Itatiba: Programa de Pós-Graduação Stricto Sensu em Psicologia da Universidade São Francisco.

Féres-Carneiro, T. (1992). Família e saúde mental. Psicologia: Teoria e Pesquisa, 8, suplemento, 485493.

Ferster, C. B., Culbertson, S. \& Boren, M. C. (1977). Depressão clínica. Em C. B. Ferster, S. Culbertson \& M. C. Boren. Principios do comportamento (M. I. Silva, M. A. Rodrigues, \&
M. B. Pardo, Trans., pp. 699-725). São Paulo: Hucitec.

Goreinstein, C.; Andrande, L. H. S. G. \& Zuardi, A. W. (2000). Escalas de Avaliação Clínica em Psiquiatria e Psicofarmacologia. São Paulo: Lemos.

Grevet, E. H., Knijnik, L. (2001). Diagnóstico de depressão maior e distimia. Revista AMRIGS, Porto Alegre, 45(3,4), 108-110.

Lidchi, V. \& Eisenstein, E. (2004). Adolescentes e Famílias no Contexto Médico. Em J. Mello Filho, Doença e família (pp. 217-231). São Paulo: Casa do Psicólogo.

Luecken, L. J. (2000). Attachment and loss experiences during childhood are associated with adult hostility, depression, and social support. Journal Psychosomatic Research, 49, 8591.

Martin, G. \& Waite, S. (1994). Parental Bonding and Vulnerability to adolescent suicide. Acta Psychiatrica Scandinávica, 89, 246-254.

Ostrander, R., Weinfurt, K. P. \& Nay, W. R. (1998). The role of age, family support, and negative cognitions in the prediction of depressive symptoms. School Psychology Review, 27 (1), 121 137.

Organização Mundial de Saúde (OMS) (2001). Relatório Mundial da Saúde: Saúde mental: nova concepção, nova esperança. Recuperado em 20 de nov.

2005: http://www.who.int/whr/2001/en/whr01_po. pdf.

Parker, G. (1979). Parental characteristics in relation to depressive disorders. British Journal of Psychiatry, 134, 138-47.

Parker, G., Tupling, H., \& Brown, L.B. (1979). A parental bonding instrument. British Journal of Medical Psychology, 52, 1 - 10.

Patten, S. B. (1991). The loss of a parent during childhood as a risk factor for depression. Canadian Journal Psychiatry, 36, 706-11.

Prieto, G., \& Mufliz, J. (2000). Um modelo para evaluar la calidad de tests usados na Espanha. Obtido em março de 2008, em World Wide Web, http:/ / www.cop.es/vernumero.asp? $\mathrm{id}=41$

Riggoto, D. M. (2006). Evidências de validade entre suporte familiar, suporte social e autoconceito. Dissertação de Mestrado. Itatiba: Programa de Pós-Graduação Stricto-Sensu em Psicologia da Universidade São Francisco. 
Santor, D. A., Gregus, M., \& Welch, A. (2006). Eight Decades of Measurement in Depression. Measurement, 4(3), 135-155.

Santos, T. M. M. (2005). Evidência de Validade entre Percepção de Suporte Familiar e Traços de Personalidade. Dissertação de Mestrado. Itatiba: Programa de Pós-Graduação Stricto Sensu em Psicologia da Universidade São Francisco.

Souza, M. S.(2007). Suporte Familiar e Saúde Mental: evidência de validade baseada na relação com outras variáveis. Dissertação de
Mestrado. Itatiba: Programa de Pós-Graduação Stricto-Sensu em Psicologia da Universidade São Francisco.

Weinman, M. L., Buzi, R, Smith P. B., \& Mumford, D. M. (2003). Associations of family support, resiliency, and depression symptoms among indigent teens attending a family planning clinic. Psychological Reports, 93 (1), 719-31.

Recebido em abril de 2008 Reformulado em setembro de 2008 Aprovado em outubro de 2008

Sobre os autores:

Makilim Nunes Baptista é Docente e Coordenador do Laboratório de Avaliação Psicológica em Saúde Mental (LAPSAM-III) do Programa de Pós- Graduação Stricto-Sensu em Psicologia da Universidade São Francisco - USF/Itatiba/São Paulo/Brasil; Doutor pelo Departamento de Psicologia Médica e Psiquiatria da Escola Paulista de Medicina - Universidade Federal de São Paulo. Bolsista Produtividade pelo CNPq.

Mayra Silva de Souza e Doutoranda do Programa de Pós-Graduação Stricto Sensu em Psicologia da Universidade São Francisco (USF) - Itatiba/SP; Bolsista CAPES.

Gisele Aparecida da Silva Alves é Graduanda em Psicologia na Universidade São Francisco. (USF) Itatiba/SP. Bolsista PROBAIC-USF do PIC (Programa de Iniciação Científica). 